\title{
Role of the Macromolecular Architecture of Copolymers at Layer-Layer Interfaces of Multilayered Polymer Films: A Combined Morphological and Rheological Investigation
}

Bo Lu ${ }^{\mathrm{a}}$, Arnaud Bondon ${ }^{\mathrm{b}}$, Ibtissam Touil ${ }^{\mathrm{b}}$, Huagui Zhang ${ }^{\mathrm{c}}$, Pierre Alcouffe ${ }^{\mathrm{d}}$, Sébastien Pruvost ${ }^{\mathrm{b}}$, Chuntai Liu ${ }^{\mathrm{a}}$, Abderrahim Maazouz ${ }^{\mathrm{b}, \mathrm{e}}$, Khalid Lamnawar $^{\mathrm{b}, *}$

${ }^{a}$ Key Laboratory of Materials Processing and Mold (Ministry of Education), National Engineering Research Center for Advanced Polymer Processing Technology, Zhengzhou University, Zhengzhou 450002, China

${ }^{b}$ Université de Lyon, CNRS, UMR 5223, Ingénierie des Matériaux Polymères, INSA Lyon, F-69621, Villeurbanne, France

${ }^{c}$ College of Chemistry and Materials Science, Fujian Key Laboratory of Polymer Science, Fujian Normal University, Fuzhou 350007, China

${ }^{d}$ Université de Lyon, CNRS, UMR 5223, Ingénierie des Matériaux Polymères, Université Claude Bernard Lyon 1 (UCBL), F-69622, Villeurbanne, France

${ }^{e}$ Hassan II Academy of Science and Technology, 10100 Rabat, Morocco

${ }^{*}$ Corresponding author:

khalid.lamnawar@insa-lyon.fr (K. Lamnawar) 
Table S1. Atomic compositions obtained from XPS spectra for various samples

\begin{tabular}{lcccccc}
\hline \multirow{2}{*}{ Samples } & \multicolumn{4}{c}{ Atomic compositions (\%) } & \multicolumn{2}{c}{ Atomic ratio } \\
\cline { 2 - 7 } & $\mathrm{C}$ & $\mathrm{O}$ & $\mathrm{N}$ & $\mathrm{F}$ & $\mathrm{Si}$ & $\mathrm{O} / \mathrm{C}$ \\
\hline PP-g-MA(-EVOH) $^{a}$ & 82.8 & 16.6 & 0.2 & 0.3 & 0.1 & 0.20 \\
PP-g-MA(-PA6) $^{b}$ & 85.1 & 8.7 & 5.5 & 0.3 & 0.5 & 0.10 \\
PP-g-MA & 95.3 & 1.0 & 0.8 & 2.9 & $<0.1$ & 0.14 \\
EVOH & 69.0 & 16.8 & 0.5 & 13.1 & 0.6 & 0.24 \\
PA6 & 77.0 & 11.4 & 11.6 & - & - & 0.15 \\
\hline
\end{tabular}

${ }^{a}$ Unreacted EVOH chains at PP-g-MA/EVOH interface were extracted.

${ }^{b}$ Unreacted PA6 chains at PP-g-MA/PA6 interface were extracted.

Table S2. Compositions of main components of the C1s signals from XPS spectra

\begin{tabular}{lcccc}
\hline \multirow{2}{*}{ Samples } & \multicolumn{4}{c}{ Compositions (\%) } \\
\cline { 2 - 5 } & C-C/C-H & C-O/C-N & O-C-O/C=O & O-C=O \\
\hline PP-g-MA(-EVOH) $^{a}$ & 80 & 18 & 2 & $<1$ \\
PP-g-MA(-PA6) $^{b}$ & 88 & 6 & 5 & $<1$ \\
PP-g-MA & 99 & 1 & - & - \\
EVOH & 76 & 21 & - & - \\
PA6 & 67 & 17 & 17 & - \\
\hline
\end{tabular}

${ }^{a}$ Unreacted EVOH chains at PP-g-MA/EVOH interface were extracted.

${ }^{b}$ Unreacted PA6 chains at PP-g-MA/PA6 interface were extracted. 

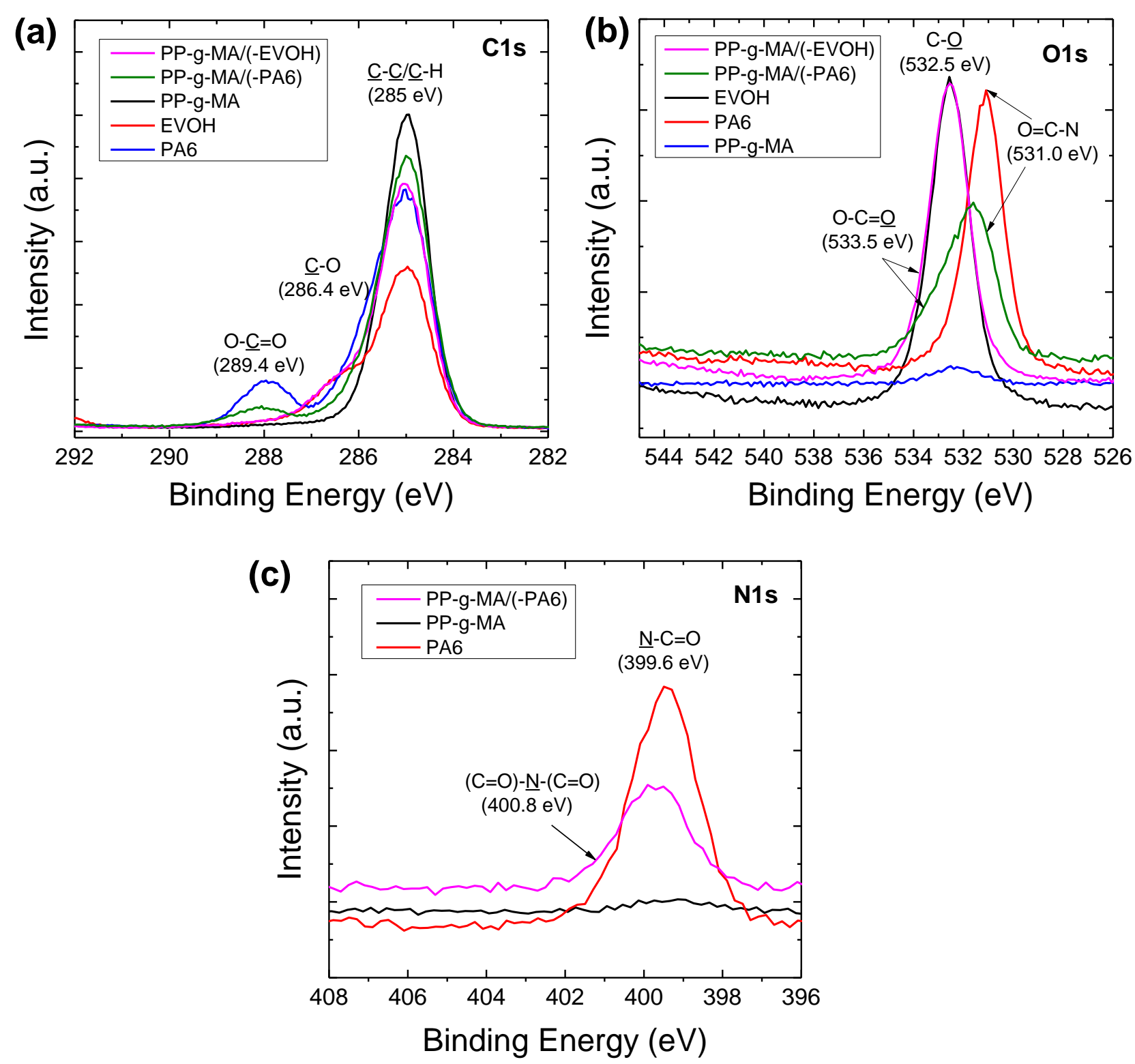

Figure S1. Comparison of XPS spectra (a) C 1s, (b) O 1s and (c) N 1s for reacted PP-g-MA/PA6 and PP-g-MA/EVOH bilayered systems after 5 min reaction and the extraction of PA6 and EVOH, and neat polymers. 

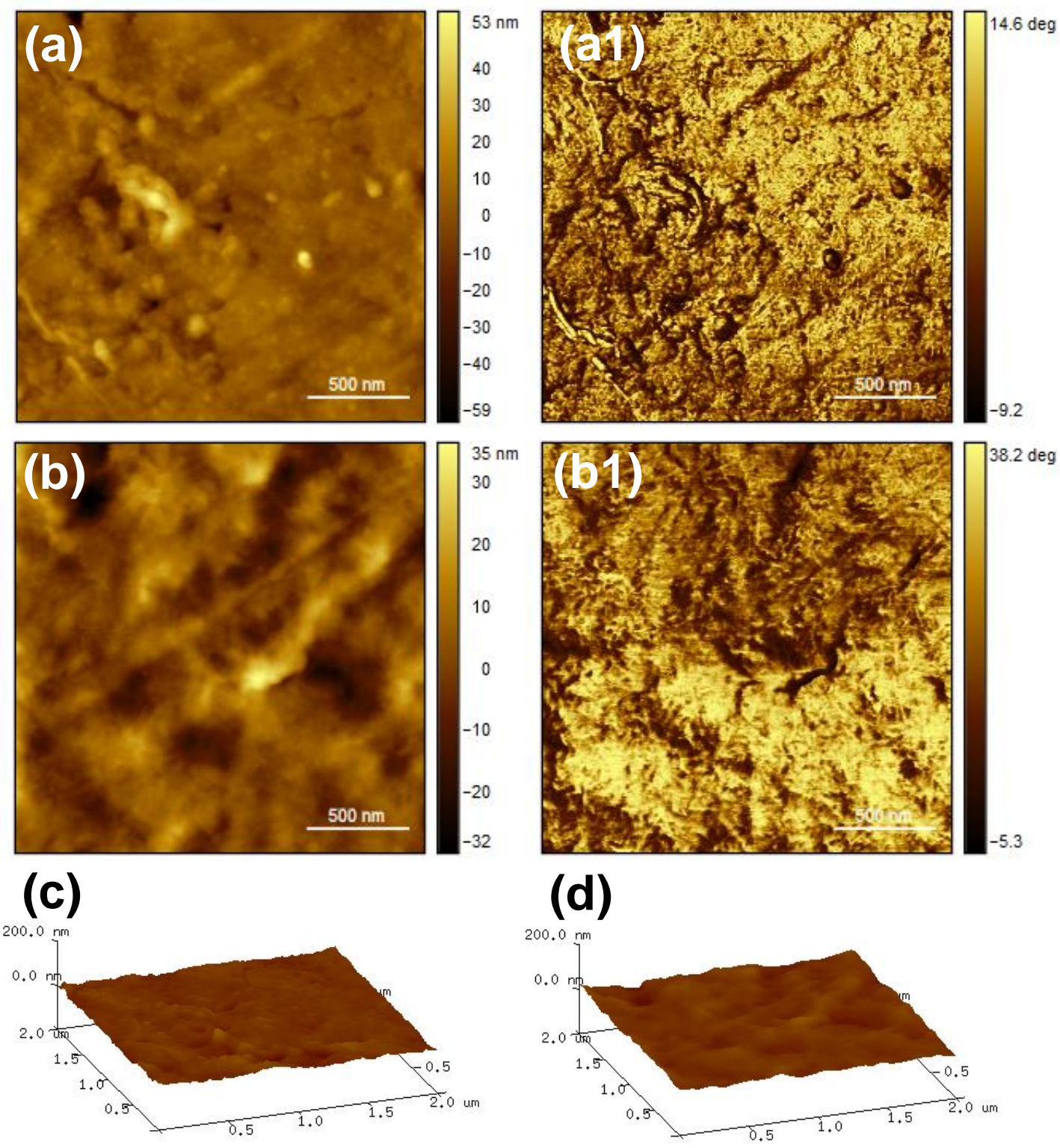

Figure S2. AFM height (a,b), phase (a1,b1), and 3D topological (c,d) images of interfaces for (a, a1 and c) PP/PA6 and (b,b1 and d) PP /EVOH reacted bilayers after the extraction of PA6 or EVOH. 